\title{
Database Integration and the Web Portal Development for the IMIRS 3D Reconstruction Package
}

Wei Dai ${ }^{1,2}$, Yuyao Liang ${ }^{2}$ and Z. Hong Zhou ${ }^{1,2}$

${ }^{1}$ School of Health Information Sciences and ${ }^{2}$ Department of Pathology and Laboratory Medicine, Medical School; University of Texas Health Science Center at Houston 6431 Fannin, Houston TX 77030

\section{Integration of an image database in data processing of IMIRS package}

Image Management and Icosahedral Reconstruction System (IMIRS) is a comprehensive suite of modular, high performance programs for three-dimensional (3D) reconstruction of icosahedral viruses and macromolecule complexes from electron cryomicroscopy (cryoEM) images [1]. One distinctive feature of the IMIRS package is that a relational image database is incorporated into the innermost layer of the package for data storage, processing and management (Fig. 1a). The modular data processing applications and accessory tools under the graphic user interface (GUI) communicate with the SQL server so that SQL programs (stored procedures) for data entry or extraction are executed on the sever side automatically. The data retrieved from one or multiple database tables are then transferred back to the modular programs for further processing and 3D reconstruction. The advantages of the integration of the database with the data processing and management are systemic data management across multiple tables, data integrity, automatic data retrieval based on user input values, and simplified user-program interaction in the complicated reconstruction. Using the integrated system, the entire $3 \mathrm{D}$ reconstruction process takes as few as only four clicks if a template (or model) is already available.

\section{Development of a web portal for IMIRS database}

To further extend the usability of the IMIRS database and to monitor the progress of data processing from anywhere at any time, we designed a web portal using active server pages (ASP) for data query, entry, and report (Fig. 1c). Four data entry forms have been designed which allow data entry while the cryoEM micrographs are recorded. Five reports with multiple levels of data abstraction were constructed to allow automatic generation of pre-designed reconstruction project summaries. In addition, four query pages were created to allow novice users to search the database with user-defined values. The web-based query and report tools also allow the display of binary images stored in the database and the seamless manipulation within a web browser. With these web-based tools, researchers with limited database knowledge can easily access data and perform those data query and entry tasks that are otherwise only performed by more advanced database users.

\section{References:}

○ [1] Y. Liang et al., J. Struct. Biol. 137 (2002) 292-304.

$\circ$ [2] This research is supported in part by US Public Health Services (AI46420 \& CA94809 to ZHZ) and the Robert Welch Foundation (AU-1492 to ZHZ). ZHZ is a Pew Scholar of Biomedical Sciences and a recipient of an Established Investigator award from the American Heart Association (\#0240216N). 
a

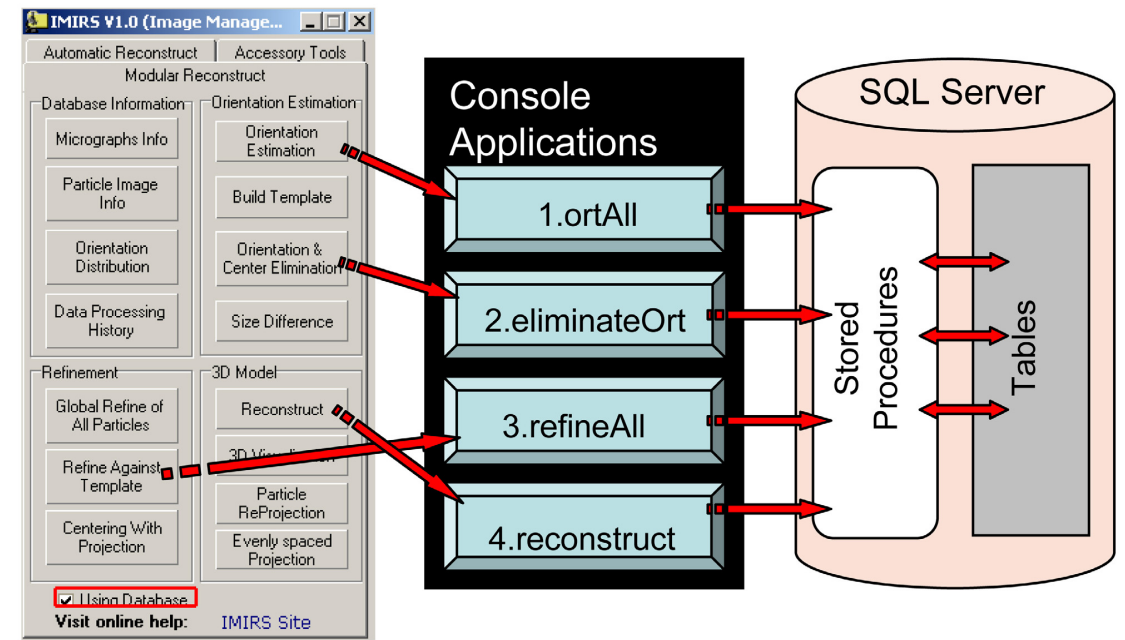

$\mathrm{b}$

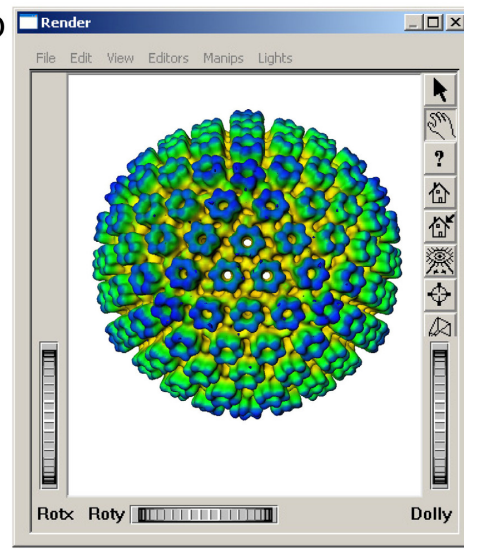

C

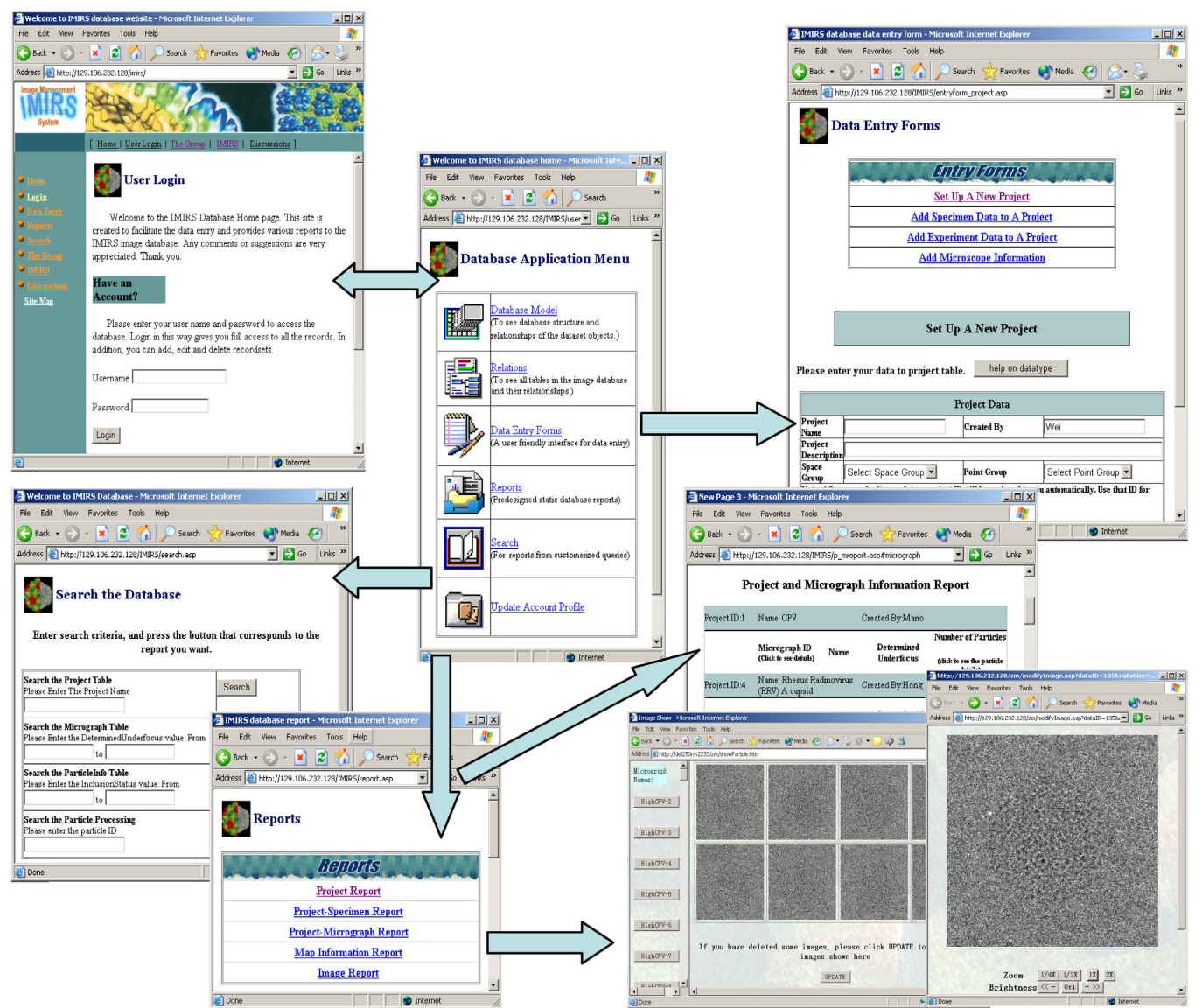

Fig.1 Database integration and the web portal development for the IMIRS reconstruction package. (a) Relationships of IMIRS GUI, console applications and the image database in IMIRS package. (b) A shaded surface representation of a herpesvirus capsid reconstructed using IMIRS GUI and database tools. (c) Representative screen shots of the IMIRS web portal, including user login, data entry forms, reports and search page. Arrows indicate the navigation steps to the screens shown. 